\title{
Comunidad. Esbozo de una historia conceptual *
}

\author{
AXEL HONNETH \\ Universidad de Frankfurt
}

Tras presentar una somera panorámica de la evolución experimentada por la noción de "comunidad" desde Aristóteles hasta Hegel, este trabajo se detiene a analizar la célebre distinción acuñada por Ferdinand Tönnies entre sociedad y comunidad. Mientras en Europa se tergiversaron enfoques teóricos como el de Tönnies hasta propiciar el surgimiento de auténticas aberraciones políticas, al contribuir involuntariamente a la creación del mito de la «comunidad del pueblo», en los Estados
Unidos el debate sobre la "comunidad" siempre representó un ingrediente indispensable para una mejor comprensión de la sociedad liberal. Aun cuando, a partir de la Segunda Guerra Mundial, el concepto de «comunidad» brilló por su ausencia en las reflexiones de la teoria política, durante los últimos años la discusión en torno al «comunitarismo» ha estimulado una inesperada revitalización del mismo y su presencia en toda suerte de consideraciones morales, políticas o sociológicas.

El hecho de que dentro del ámbito lingüístico alemán resulte bastante arduo perfilar el sentido de las discusiones filosófico-sociales, en cuyo centro se sitúa la idea de comunidad, responde a determinados condicionamientos históricos. En ninguna otra constelación de tradiciones se ha llegado a vincular con el concepto de comunidad unas cargas ideológicas tan acentuadas y aberrantes en términos políticos. Desde que Ferdinand Tönnies distinguiera dos tipos de integración social con las categorías de «comunidad» y «sociedad», para poder analizar el proceso de la modernización social bajo el punto de vista de la progresiva disolución de la solidaridad institucionalizada, este dualismo conceptual se convirtió en toda Europa, pero sobre todo en Alemania, en el punto neurálgico de un diagnóstico filosófico válido para toda una época.

Los representantes de la izquierda influida por el romanticismo, como el joven Lukács o Walter Benjamin, apenas vislumbraron en el surgimiento de la sociedad capitalista el peligro de una pérdida de las experiencias y los modos de vida ligados a la hipótesis de la existencia de sociedades éticamente integradas; al igual que quienes abogan por una crítica conservadora de la cultura tratan de aclarar el proceso de la modernización como el patológico avance del desarraigo y la masificación de lo particular, para terminar recomendando una vuelta hacia formas tradicionales de la vida en común.

\footnotetext{
* Versión castellana de Roberto R. Aramayo y Juan Carlos Velasco.
} 
Ni siquiera la perspicaz e impresionante exposición de los Límites de la comunidad en términos normativos, realizada por Helmut Plessner (1981) desde un espíritu liberal para prevenimos contra la tendencia de acrecentar los rasgos comunitarios en detrimento del ámbito social, pudo evitar que dicho concepto se viera ulteriormente sobrecargado con el explosivo material de las esperanzas y las nostalgias políticas. De tal manera que, finalmente, la categoría de comunidad se ve totalmente despojada de su contenido sociológico para quedar convertida en un Leitmotiv idcológico del nacionalsocialismo, que conduce hacia la representación de una identidad colectiva de los alemanes fundada biológicamente y que resulta decisiva para franquear el camino a la segregación totalitaria de cuanto sea foráneo (Dumont, 1983, caps. IV, V y VI). Todo ello coloca muy alto el umbral que debe atravesar el concepto de comunidad, dentro del ámbito lingüístico alemán, antes de poder ser aplicado a las cuestiones contemporáneas.

Ciertamente, esta barrera político-moral tampoco debe convertirse en un escollo insuperable que nos impida sempiternamente percibir los desafíos y las crisis de las distintas épocas en aquellas categorias que les corresponden. De ahi que sea conveniente repasar primero de un modo sucinto la historia conceptual de «comunidad», para examinar después en qué modo dicho concepto puede aportar actualmente una base oportuna para la reflexión moral y sociológica.

\section{I}

Hasta los ticmpos del primer romanticismo, e incluso propiamente hasta el umbral del siglo $\mathrm{xx}$, la filosofía política no ha llevado a cabo una distinción efectiva entre «comunidad» y «sociedad» (Riedel, 1975). Aristóteles, quien con su Ética a Nicómaco asentó las bases teóricas de la doctrina política clásica, recogía en el concepto de koinonia todas las formas de socialización del ser humano sin difcrenciar sistemáticamente entre grados de avenencia o intensidad de los sentimientos; el hombre, que es un ser político por naturaleza, se mueve siempre en el horizonte de acción de la polis, en la que se integra socialmente conforme a los distintos tipos de asociación configurados por los diferentes estamentos y empleos. Fn estas formas de asociación predomina una u otra proporción de «amistad» y «justicia», pero por lo común sólo presentan algunos matices expresivos y resulta prácticamente indiferente utilizar los términos «comunidad» o "sociedad», por cuanto que el concepto de koinonia comprende tanto los distintos tipos de agrupaciones humanas basadas en algún acuerdo (promesa, contrato) como también las originarias formas «naturales» de la vida en común dentro del ámbito doméstico, vecinal o étnico.

Este amplio concepto de koinonia, en donde no se distingue sistemáticamente ni entre el interés o el sentimiento como argamasa del vínculo ni entre unión «natural» o «social», sigue predominando también en el pensa- 
miento del tardoclasicismo y del medioevo cristiano. Es cierto que sobre los diversos estratos de la recepción de Aristóteles su doctrina se acrecienta merced a ciertos criterios o se establecen diferencias en determinados respectos, agregándose una significación teológica o amplificándose en torno al concepto de una paulatina construcción de las sociedades, pero el núcleo de su propuesta conceptual permanece sustancialmente intacta: la koinonia sigue siendo el sinónimo aplicable a las expresiones latinas societas o communitas, en cuanto síntesis de todas las formas de una agrupación social donde los hombres se reúnan para la persecución conjunta de sus intereses o en aras de un vínculo emocional.

Una transformación sustantiva del lenguaje conceptual sólo tiene lugar en el terreno de la filosofía política cuando en los escritos de Hobbes y Spinoza va cobrando cuerpo el derecho natural moderno. Entonces comienza ese largo proceso de una ilustración filosófica en el que la naturaleza pierde progresivamente su papel como fundamento explicativo de la socialización humana, de tal modo que «comunidad" o "sociedad» ya no pueden ser entendidas como una sustancia o un fin, sino que pasan a ser definidas como una «función»; para Hobbes, a excepción de la «familia» (que a partir de ahora será concebida como la esfera de lo privado), todas las formas de un agrupamiento social (corporaciones, congregaciones, sociedades mercantiles) son presentadas como configuraciones secundarias en el marco de un ordenamiento estatal que se constituye mediante contrato.

Estos cambios conceptuales, en los que se empieza a perfilar nuestro moderno conccpto de «sociedad civil», se fijan por primera vez de un modo sistemático en la filosofía política de John Locke; éste atribuye consecuentemente a un contrato cualquier configuración social y sustituye al mismo tiempo la idea clásico-aristotélica de una finalidad tendente a perfección por la función conceptual de la seguridad (de la vida y el patrimonio). Con ello quedaba el terreno bien abonado para un sinmúmero de proyectos contractuales en los que, aun cuando no se suele distinguir todavia entre «sociedad» $\mathrm{y}$ «comunidad» de un modo sistemático, sí va cobrando cuerpo la idea jurídica tendente a clarificar el concepto de «comunidad»: en Rousseau la sociedad civil se constituye mediante un contract social donde los ciudadanos deciden, merced a un acto colectivo de conversión moral, crear una república organizada jurídicamente; para Kant el Estado de derecho se define a partir de la idea práctico-racional de una constitución cuya soberana encarnación es esa ley que permite compatibilizar la libertad de cada cual con la de todos los demás; y finalmente para Fichte (quien anticipa el ulterior distingo entre Estado y sociedad) la «sociedad» representa, en el sentido estricto del término, ese «agregado» de seres racionales libres que se encuentra habilitado para asentar un ordenamiento jurídico regulado públicamente al margen de la fuerza.

Sin lugar a dudas, todas estas conjeturas jurídico-racionales presentan enormes diferencias las unas respecto de las otras, al albergar distintos supuestos sobre las comunidades prepolíticas o morales que han de subyacer a la sociedad 
de ciudadanos libres e iguales. Ya en Rousseau se encuentra la reflexión de que la república constituida contractualmente sólo puede cobrar estabilidad cuando los miembros están culturalmente vinculados entre sí merced a una religión civil; y el último Fichte sustenta incluso la jdea de que cualquier ordenamiento estatal racionalmente regulado precisa de un enraizamiento en una comunidad prepolítica, como el que sólo puede proporcionar la experiencia de una homogeneidad "nacional». Esta suerte de meditaciones es a la que pueden referirse los modelos sociales del movimiento romántico para delimitar, si no el concepto, sí la idea de que la sociedad moderna constituye una unión social conforme al prototipo de la «comunidad»: mientras que los individuos se atienen por ello al comportamiento jurídico de la sociedad, de tal modo que persiguen recíprocamente sus intereses o fines establecidos, se ven mutuamente vinculados on las asociaciones familiares, vecinales y étnicas que van formándose merced a lazos prerracionales, como aquellos que nacen a partir del afecto, de los usos y de las interdependencias.

Para caracterizar esta peculiar forma de socialización se encuentran en la terminología política del romanticismo alemán expresiones deudoras de la traducción de las Reflections on the Revolution in France (1793) -el célebre texto de Edmund Burke. Así, la idea de una asociación que se ha formado en oposición a una relación contractual meramente histórica y une a varias generaciones en virtud de una armonización de intereses, viene a traducirse unas veces con el concepto del "vínculo" social, pero en otras ocasiones es expresada recurriendo al concepto de «comunidad».

Al elaborar su sistema, Hegel intenta reunir en un único principio las diversas aportaciones de la tradición que inopinadamente coexisten a comienzos del siglo xIx; con ello no sólo se convierte en el precursor de la teoría materialista de la sociedad, sino que también es el pionero de la sociología establecida hacia el final de ese mismo siglo (Hegel, 1970). Para Hegel toda forma de unión social (que en Locke, Rousseau o Kant era concebida según el modelo de una relación contractual como sociedad de ciudadanos libres e iguales) sólo representa una de las tres esferas de la sociedad moderna: junto al «sistema de las necesidades», como designa también Hegel a la primaria función económica del ámbito comercial constituido jurídicamente, coloca por una parte a la esfera privada de la familia y, de otro lado, le antepone esa esfera del Estado que concibe de una manera que raya el sustancialismo.

No cabe reconstruir aquí el trasfondo de la compleja meditación histórico-conceptual que le permite a Hegel arribar hasta esta tripartición categorial; pero incluso una somera ojeada evidencia que quiso tener en cuenta todos los conceptos de sociedad o de comunidad desarrollados hasta entonces, al intentar comprender a la "sociedad civil» según el modelo contractual moderno, a la «familia» conforme al modelo romántico de la asociación y finalmente al «Estado» con arreglo a la comprensión aristotélica de la koinonia. Surge así una teoría merced a la cual la sociedad moderna entraña tres formas de 
socialización que se diferencian entre sí en virtud de su respectivo grado de avenencia e intensidad afectiva: mientras que dentro de la «familia» los sujetos se ven ligados por la fuerza de los lazos del amor mutuo, en la esfera económica de la "sociedad civil» sólo están vinculados por la «libertad negativa» de las relaciones contractuales $y$, a la postre, lo único que les mantiene unidos en el «Estado» es el compromiso compartido de un fin superior.

Con esta compleja teoría social Hegel contribuye decisivamente a preparar el terreno para la ulterior distinción entre «comunidad» y «sociedad», aun. cuando él mismo no aplique de un modo sistemático ese dualismo conceptual; sin embargo, por muy rica en consecuencias que haya resultado ser su tripartición, por mucho que haya podido influir en la formación del marxismo y de las ciencias sociales, era obvio desde un principio lo que constituiría su problema central en el futuro: icómo debería entenderse aquel «fin superior» por medio del cual se relacionan entre sí de un modo positivo los sujetos dentro del Estado, si la tendencia evolutiva de la sociedad moderna debía orientarse hacia una mayor pluralización de los valores y una marcada individualización de los estilos de vida?

\section{II}

Cuando en los años ochenta del siglo pasado Ferdinand Tönnies emprendió la redacción de su libro Comunidad y sociedad (Tönnies, 1992) pudo sustentarse en una pluralidad de innovaciones conceptuales y perspectivas teóricas que había propiciado el desarrollo de la teoría social posterior a Hegel. En suma, al tocar a su fin la época del idealismo alemán se produjo un giro «empírico» en la formación de la teoría político-práctica que condujo a que se colocara paulatinamente en el centro de atención la cuestión del origen histórico y la cstructura institucional de las sociedades industriales modernas.

Desde un talante liberal, Lorenz von Stein ya había propuesto poco tiempo después de la muerte de Hegel concebir la «comunidad» como aquella esfera en la que los individuos viven los unos para los otros, de tal modo que las contraposiciones de intereses de la sociedad industrial pudieran ser superadas; Marx, polemizando críticamente contra Hegel, afrontó la cuestión de cómo el modo capitalista de la economía podía conducir a una forma de sociedad en donde todas las formas comunitarias o cooperativas entre los sujetos hubieran sido destruidas por la competencia y la presión del beneficio; y Herbert Spencer, finalmente, había explicado el tránsito desde una sociedad tradicional a una sociedad moderna, con la ayuda de una distinción entre dos formas jurídicas históricamente consecutivas en donde las pretensiones jurídicas individuales se derivan unas veces del «status» otorgado o adquirido y otras de un «contrato» suscrito voluntariamente.

Se necesitaría probablemente un señalado talento sintético para alcanzar mediante estas variadas aportaciones y reflexiones la distinción conceptual que 
había aportado el título del libro de Tönnies. Debe denominarse «comunidad» a aquella forma de socialización en la que los sujetos, en razón de su procedencia común, proximidad local o convicciones axiológicas compartidas, han logrado un grado tal de consenso implícito que llegan a sintonizar en los criterios de apreciación; mientras que con «sociedad» se alude a aquellas esferas de socialización en donde los sujetos concuerdan en consideraciones racionales ajustadas a fines, con el objeto de obtener la reciproca maximización del provecho individual.

En los planteamientos de Tönnies no quedaba muy claro si a esta distinción le correspondía únicamente un valor analítico o también uno empírico, pero sí era enormemente ilustradora y provocativa la tesis relativa al diagnóstico de toda una época que pretendia desarrollar con su ayuda, a saber: que en la puesta en marcha de la sociedad capitalista las esferas «sociales» de acción reprimen o diluyen paulatinamente aquellas relaciones sociales que poseen el carácter distintivo de las comunidades. De todas formas, este diagnóstico de Tönnies no fue conccbido como una tesis de filosofía de la historia que debiera afirmar la irreversibilidad o inevitabilidad de una determinada tendencia evolutiva; ni tampoco pretendía oficiar como una suerte de romántico social que de modo meramente nostálgico anhela arcaicas formas de la vida comunitaria del mundo rural. Antes bien, la totalidad del compromiso del socialdemócrata Tönnies iba encaminado a la tarea de explorar las posibilidades sociales de crear comunidades tales que, como las corporaciones o los sindicatos, se adecuasen a las condiciones de la era industrial.

Incluso los esfuerzos conceptuales que tan sólo una década después emprende Durkheim, y que se hallan orientados hacia una diferenciación sistemática entre dos formas de integración social, sirven ante todo al objetivo de solventar la crisis moral de la sociedad industrial (Durkheim, 1977). Aun cuando es cierto que no emplea el par conceptual de «comunidad» y «sociedad», en una crítica al escrito de Tönnies, que ha devenido célebre, sí llega a prevenirnos respecto de las tergiversaciones político-culturales que pueden cernirse sobre semejante disquisición; sin embargo, aquellas precisiones conceptuales que él mismo propone posteriormente para delimitar la solidaridad «mecánica» de la "orgánica» vienen a coincidir en lo esencial con las propuestas conceptuales de Tönnies: mientras que bajo las condiciones de la «solidaridad mecánica» predomina entre los sujetos un grado tan elevado de armonía emocional y cognitiva que la integración social puede llevarse a cabo sobre la estable base de una conciencia colectiva, bajo las condiciones de la «solidaridad orgánica» las diferencias individuales entre los sujetos son tan enormes que tan sólo la coacción cooperativa de la división del trabajo puede proporcionar integración social.

Pero, mientras que Tönnies con su diferenciación conceptual quería, ante todo, llamar la atención sobre dos formas de socialización que han de alcanzar alguna suerte de equilibrio en la sociedad moderna, Durkheim parte sin ambages 
de una consecuencia evolutiva de las dos formas de solidaridad: bajo la presión de un proceso permanente de individualización, como sucede con la transformación estructural del derecho penal y los modificados modelos de la conciencia religiosa, la forma mecánica de solidaridad es reemplazada paulatinamente por los mecanismos integradores de la división del trabajo. Éste es un nuevo tipo de integración social en el que Durkheim advierte el problema epocal a cuya resolución ha de contribuir con sus estudios sociológicos; pues algo de lo cual estuvo convencido durante toda su vida es que, sin mecanismos correctivos o supletorios, la integración conduce mediante la división del trabajo a un estado social en el que se concita una peligrosa carencia de acuerdos morales básicos entre los sujetos.

Como terapia para esta amenazadora situación se brinda la propuesta, presente ya en los primeros escritos de Durkheim, de reorganizar «justa» o «equitativamente» la división del trabajo de tal manera que todos los miembros de la sociedad puedan saberse ligados moralmente al objetivo común; por tanto, ésta debe ser la nueva forma de integración que proporcione los recursos normativos con los cuales se crea cl grado necesario de vínculos morales entre los sujetos. Pero, decepcionado por los resultados prácticos de su propuesta, en los últimos años de su vida Durkheim da un viraje hacia una idea que comporta rasgos mucho más radicales: ahora ya no es la justa reorganización de la división del trabajo, sino la periódica repetición de situaciones proclives a una fusión colectiva, lo único que puede conducir en las sociedades modernas hacia una forma de integración social que no comporte el peligro de desorganización moral. Con cste planteamiento teórico procedente de su libro sobre la religión Durkheim se convirtió también, sin quererlo y no de un modo muy diferente que Tönnies, en el instigador de un concepto de comunidad que no mucho más tarde se vería pertrechado con la matcria inflamable del resentimiento crítico-cultural.

A dicha politización del concepto de comunidad pudo llegarse, tanto en Alemania como en Francia, en el momento en que con el anticapitalismo romántico y la crítica cultural conservadora surgieron dos corrientes intelectuales que descubren en la expansión del capitalismo el peligro de una mecanización y atomización de la vida social: con «comunidad» se designa, enlazando tenuemente con Tönnies o con el Durkheim tardío, aquella forma de unión social en la que los sujetos, en virtud de puntos en común generados históricamente o provistos biológicamente, mantienen entre sí unos lazos afectivos más fuertes que los habituales en las meras relaciones jurídicas; como ejemplos ilustrativos de tales «comunidades" valen la familia, las poblaciones rurales medievales o las sectas religiosas (Löwy/Sayre, 1983). Tampoco el escrito injustamente olvidado de Helmut Plessner, con el que se introdujo una definición liberal de los Límites de la comunidad, pudo impedir que esta retrógrada utopía de la comunidad generada orgánicamente pudiera hallar rápidamente la más amplia resonancia en los ámbitos intelectuales; en la izquierda, la «comunidad» 
se erigió en el concepto polémico para la creación militante de una clase obrera politizada; en la derecha, por el contrario, se convirtió en el concepto polémico para la realización política de una «comunidad del pueblo» que ya no estaba vinculada al marco del Estado de derecho propio de la sociedad liberal (Plessner, 1981).

Ahora bien, aun cuando el concepto de la "comunidad" se había erigido no sólo en la contraposición categorial, sino también política, de la idea liberal de sociedad, la evolución conceptual en los Estados Unidos durante el mismo período de tiempo tomó un rumbo completamente distinto (Joas, 1993; Bender, 1995). Aquí la discusión sobre la «comunidad» supuso desde el principio un elemento constitutivo de la autocomprensión política de una sociedad liberal; se consideró la cuestión central hasta qué punto la sociedad constituida democráticamente podía quedar privada de toda vinculación con las communities locales o religiosas sin perder sus propios presupuestos existenciales. Esta especial situación de partida tuvo como consecuencia que también la recepción del par conceptual propuesto por Tönnies se adentrara en los Estados Unidos por unos derroteros diferentes que en Alemania o Francia. Si bien el texto en cuanto tal apenas había ejercido ninguna influencia sobre el pensamiento norteamericano, la distinción entre «comunidad» y «sociedad» sería entendida en el sentido no de un esquema bifásico, sino trifásico: a la pérdida de las formas originarias de comunidad no le sigue la expansión desenfrenada de esferas «sociales», sino una ola de creación de nuevas comunidades, a menudo sustentadas en las culturas de los países de procedencia de los inmigrantes, que posteriormente se encuentran siempre amenazadas por las tendencias atomizadoras de la sociedad. Esta cándida recepción, ni nostálgica ni culturalmente crítica, del concepto sociológico de «comunidad» permitía concebir el establecimiento de una sociedad democrática incluso como un proyecto «comunitarion en el que participasen activamente las diferentes communities: desde John Dewey hasta John Rawls se extiende en los Estados Unidos la tradición de una filosofía política en la que la democracia establecida en términos de Estado de derecho fue concebida como una community of communities (Dewey, 1946). De cste modo nació en esta reinterpretación liberal, y de una manera casi imperceptible para la inteligencia europea, un concepto de "comunidad" como quizás a Tönnies le hubiera gustado desarrollar en su malograda orientación hacia el movimiento cooperativo: deben llamarse communities a esas formas de unión social en las que los sujetos articulan por la vía de la participación democrática valores y metas hacia los que se sienten vinculados colectiva e igualitariamente. 
Despućs de que, tras la Segunda Guerra Mundial y por motivos político-morales, el concepto de «comunidad» desapareciera prácticamente del lenguaje de la escena pública intelectual, el debate sobre cl «comunitarismo» le ha ayudado a obtener en los últimos años un renacimiento inesperado; es de temer que en el ínterin se vincule nuevamente con él una pluralidad tal de propósitos teóricos y expectativas políticas que, a lo largo de la controversia, llegue a perder cualquier significado claramente perfilado. En efecto, el concepto vivirá en adelante, como ya lo hizo en la época del movimiento romántico, cuando fue empleado por primera vez de manera consciente como un término crítico, de su contraposición apelativa, e incluso polémica, con respecto del concepto moderno de «sociedad»: mediante este término se piensa desde Locke, Kant o Hegel en una forma de unión social en la que los sujetos se respetan mutuamente como portadores individuales de derechos, del mismo modo que «comunidad» debe designar un tipo de asociación en la que los sujetos mantienen de alguna manera lazos positivos con los demás. Pero en qué consisten estos lazos o si éstos deben proceder de sentimientos compartidos, convicciones comunes o recuerdos históricos, son cuestiones tan poco claras en la discusión contemporánea como la de cuán profundos deben ser aquellos lazos en el sujeto individual o si éste puede ser miembro de distintas comunidades o de manera permanente sólo puede ser parte integrante de una única comunidad constitutiva. Sin duda, si se considera con mayor atención el contexto teórico en el que hoy en día se habla en un sentido específico de «comunidad», entonces se perfilan tres usos diferentes de dicho concepto:

a) En el contexto de la filosofía moral, el concepto asume en nuestros días sobre todo la función de llamar la atención sobre un estrato previo de valores comúnmente compartidos que en la fundamentación de normas o principios morales no resultan fácil de soslayar o poner entre paréntesis; antes bien, toda fundamentación permanece ligada necesariamente a un horizonte de convicciones axiológicas compartidas, porque gracias a ello queda ya establecido en qué consiste en cada caso la idea de la moral. Aquí, con el concepto de «comunidad» se pondera aquel elemento que, en el sentido del modelo hegeliano de la eticidad (Sittlichkeit), subraya que los sujetos en la vida social también pueden estar ligados unos con otros por medio de orientaciones axiológicas comunes; con mucha frecuencia no resulta nada claro si con esto se está pensando en una necesidad trascendental o en una posibilidad históricamente contingente.

b) En el contexto de la sociología, el concepto asume en nuestros días principalmente la función de llamar la atención sobre las posibilidades de formación de grupos solidarios mediante los cuales los sujetos puedan eludir el 
peligro de aislamiento social. Con ello se está presuponiendo a menudo un proceso histórico de individualización creciente que sólo debe ser contrarrestado posteriormente, si pueden ser abiertas nuevas fuentes del vínculo intersubjetivo o si pueden ser revitalizadas las viejas fuentes como, por ejemplo, las religiosas. Aquí se pondera, por lo tanto, en el concepto de «comunidad» aquel elemento que, en el sentido de Durkheim o de John Dewey, subraya que los sujetos precisan del sostén incuestionado de un grupo estimulante o intercesor con el objeto de asegurar la identidad; en este orden de cosas queda sin aclarar cuán amplios pueden o deben ser tales grupos actualmente, a la vista del creciente pluralismo axiológico.

c) En el contexto político, el concepto tiene ante todo la función de llamar la atención sobre aquellas formas de participación comunitaria que deben formar parte de las condiciones de una democracia vital; el punto de partida es que las posibilidades de una participación tal aumentan en la medida en que los sujetos pucdan saberse vinculados activamente a una meta común. Aquí, en el concepto de "comunidad» se pondera, pues, aquel elemento que, en el sentido del pragmatismo americano, subraya que entre el vínculo axiológico común y la participación activa existe una relación de refuerzo mutuo; hoy en día, no resulta claro, de todas mancras, cuál es la fucnte de donde deben proceder aquellos valores comunes con los que poner en marcha el intercambio entre vínculo comunitario y participación.

\section{REFERENCIAS BIBLIOGRÁFICAS}

Bender, T. (1995): «Community», en Richard Wightmann Fox y James T. Kloppenberg (eds.), A Companion to American Thought, Oxford, pp. 137-140.

Dewey, J. (1946): The Public and its Problems. An Essay in Political Inquiry, Chicago.

Dumont, L. (1983): Essais sur lindividualisme. Une perspective anthropologique sur lideologie modene, París (Ensayos sobre el individualismo. Una perspectiva antropológica sobre la ideologia moderna, Madrid, Alianza, 1987).

Durkheim, E. (1930): De la division du travail social, París (La división del trabajo social, Madrid, Akal, 1987).

Hegel, G. W. F. (1970): Grundlinien der Philosophie des Rechts (Fundamentos de la Filosofia del Derecho, Madrid, Libertarias/Prodhufi, 1993).

JOAS, H. (1993): "Gemeinschaft und Demokratie in den USA. Die vergessene Vorgeschichte der Kommunitarismus-Diskussion», cn Micha Brumlik y Hauke Brunkhorst (eds.), Gemeinschaft und Gerechtigkeit, Francfort.

LöWY, M., y SAYRE, R. (1983): «Figures du romantisme anticapitaliste», en L'Homme et la Société, vol. $69 / 70$, pp. 99 y ss.

Plessner, H. (1981): Grenzen der Gemeinschaft. Eine Kritik der sozialen Radikalismus (1924), en Gesammelte Schriften, vol. V, Macht und menschliche Natur (ed. por Odo Marquard, Günter Dux y Elisabeth Ströker), Francfort. 
RIEDEL, M. (1975): «Gesellschaft» y «Gemeinschaft», en Otto Brunner, Werner Conzen y Reinhart Kosseleck (eds.), Geschichtiche Grundbegriffe. Historisches Lexikon zur politisch-sozialen Sprache in Deutschland, Stuttgart.

SelzNICK, Ph. (1992): The Moral Commonwealth. Social Theory and the Promise of Community, Berkeley.

Tönnies, F. (1912): Gemeischaft und Gesellschaft, Grundbegriffe der reinen Soziologie, Berlín (Comunidad y asociación, Barcelona, Peninsula, 1979). 